\title{
Effects of Ricean Fading and Data Modulation on Noncoherent PN Sequence Sequential Acquisition Schemes
}

\author{
Alex W. Lam, Sawasd Tantaratana, and Patrick J. Vincent
}

\begin{abstract}
Power-efficient and robust sequence acquisition schemes for noncoherent direct-sequence spreadspectrum (DS/SS) communications are of interest. Fast and reliable schemes with low false-alarm and miss probabilities have been designed. In this paper, we investigate the performance of various pseudo-noise (PN) sequence acquisition schemes with data modulation in a Ricean fading channel. In particular, the fixed sample size test (FSST) and the truncated sequential probability ratio test (TSPRT) are studied in detail. In previous studies of sequential acquisition tests, the integrators in the correlation process are reset periodically to generate i.i.d. samples; unfortunately, this also introduces loss in the effective signal-to-noise ratio and hence degrades the system performance. We do not reset the integrators and our decision samples are neither independent nor identically distributed. The probability density functions of the decision statistics are determined and the decision thresholds for achieving the design false-alarm and miss probabilities can be calculated. However, the average sample size (ASN) of a sequential test does not have a closed form analytical solution and it is evaluated by simulation.
\end{abstract}

I. Introduction

In direct-sequence spread-spectrum (DS/SS) communications, synchronization of the incoming SS signal and the local generator output can be achieved in two stages: acquisition and tracking. First, the acquisition mechanism attempts to coarsely align the incoming pseudo-noise (PN) sequence phase with the locally generated PN sequence phase. Once the two sequence phases are aligned correctly within a small range, the tracking mechanism is activated to fine-tune and maintain the perfect phase-lock. Many studies in PN acquisition are available in the literature; for example, see [1] - [8] and the references listed therein. In this work, we are interested in power-efficient and robust sequential acquisition schemes for DS/SS communications with noncoherent demodulation. In most of the studies of sequential acquisition tests, the integrators in the correlation process are reset periodically to generate i.i.d. samples; unfortunately, this introduces loss in the effective signal-to-noise ratio and hence degrades the system performance. We do not reset the integrators in our sequential schemes, consequently, our decision samples are neither independent nor identically distributed.

In [8] and [9], the noncoherent sequential probability ratio test (SPRT) and the truncated sequential probability ratio test (TSPRT) were studied and methods for designing the decision thresholds to achieve the design false-alarm probability $\left(\alpha_{d}\right)$ and miss probability $\left(\beta_{d}\right)$ were presented. Based on simulations, the average sample numbers (ASN), i.e., the mean number of samples needed before making a decision, of various

This work was supported by the Army Research Office under Grant DAAL03-91-G-0001. A. W. Lam and P. J. Vincent are with the Department of Electrical and Computer Engineering, Naval Postgraduate School, Monterey, CA 93943. Email: lam@ece.nps.navy.mil S. Tantaratana is with the Department of Electrical and Computer Engineering,University of Massachusetts, Amherst, MA 01003. Email: tantaratana@ecs.umass.edu schemes were compared. It was found that the TSPRT could be essentially as efficient as the SPRT under non-alignment (denoted as state $\mathrm{H}_{0}$ ) between the two sequences, and slightly less efficient than the SPRT under alignment (denoted as state $\left.H_{1}\right)$. In addition, the TSPRT was found to be uniformly better than the fixed-sample-size test (FSST). In this paper, we extend our investigation on sequential acquisition schemes to include Ricean fading and data modulation. We will discuss the effect of fading on the decision statistics in Section II. The effect of data modulation will be outlined in Section III. Numerical results will be presented in Section IV, and finally, some concluding remarks will be given in Section $\mathrm{V}$.

\section{Acquisition in Fading Channel}

The noncoherent acquisition system considered in this paper is depicted in Figure 1. Only a brief account of the sequential schemes will be given in here; a detailed treatment can be found in [8] and [9]. Referring to Figure 1, the received signal and noise can be written as:

$$
r(t)=A_{0} \psi a\left(t+i \Delta T_{c}\right) \cos \left(\omega_{0} t+\theta\right)+n(t)
$$

In the above equation, $A_{0}$ is the signal amplitude, $\psi$ is a fading random variable (r.v.), $a\left(t+i \Delta T_{c}\right)$ is the incoming $P N$ waveform with phase off-set $i \Delta T_{c}$, " $i$ " is an integer, $\Delta$ is a constant for the phase updating process, $T_{c}$ is the chip duration, $\omega_{0}$ and $\theta$ are the carrier frequency and phase, and $n(t)$ is the additive white Gaussian noise (AWGN) with two-sided power spectral density (PSD) equal to $N_{0} / 2$. The value of $\Delta$ is usually taken to be 1 or $1 / 2$, implying that the phase is updated each time by $T_{c}$ or $T_{c} / 2$. In Figure 1, the locally generated PN waveform is denoted as $a\left(t+(j+\gamma) \Delta T_{c}\right)$, where $" j "$ is an integer and $l l<1 / 2$. The received signal $r(t)$ is first multiplied by the locally generated PN waveform, and then followed by noncoherent demodulation. The resulting decision statistic, $Y_{n}$, is used to test that if the local and the incoming PN waveforms are aligned to within $\Delta T_{d} / 2$ seconds. When coarse alignment is achieved, we have $j=i$ and the tracking circuit is initiated to fine-tune $\gamma$ to zero. Otherwise, the phase of the local PN waveform is updated by $\Delta T_{c}$ and the acquisition process will continue.

In this paper, we consider PN acquisition performance in a Ricean fading channel. We model the fading r.v. $\psi$ in $(2.1)$ as Ricean with probability density function (p.d.f.) given by [10]:

$f(\psi)=2 \psi(1+r) e^{-r-\psi^{2}(1+r)} I_{0}(2 \psi \sqrt{r(1+r)}), \quad \psi \geq 0 \quad(2.2)$ The parameter $r$ in $(2.2)$ is the ratio of the power in the direct component to the power in the diffused component, with the normalized constraint that the sum of these two component powers is equal to 1 . If $r=\infty$, there is no fading. On the other hand, if $r=0, \psi$ is Rayleigh distributed and the fading will be the most severe. In this paper, we have assumed slow fading so that $\psi$ does not vary much within one data bit interval. The decision statistic

$$
Y_{n}=X_{i, n}^{2}+X_{q, n}^{2}
$$

is the sum of the square of the in-phase component $X_{\mathrm{inn}}$ and the square of the quadrature component $X_{q, n}$, where 
and $\quad X_{i, n}=\int_{0}^{n T_{c}} r(t) a\left(t+(j+\gamma) \Delta T_{c}\right) \cos \omega_{0} t d t$

$$
X_{q, n}=\int_{0}^{n T_{c}} r(t) a\left(t+(j+\gamma) \Delta T_{c}\right) \sin \omega_{0} t d t
$$

When $\psi=1$ (no fading), $X_{i, n}$ and $X_{q, n}$ are conditionally independent Gaussian r.v.s with variance $\sigma_{n}^{2}=n T_{c} N_{0} / 4$ and means $\left(A_{0} T_{c} S_{n} / 2\right) \cos \theta$ and $\left(A_{0} T_{c} S_{n} / 2\right) \sin \theta$, respectively. Here,

$$
\begin{aligned}
S_{n} & =\frac{1}{T_{c}} \int_{0}^{n T_{c}} a\left(t+i \Delta T_{c}\right) a\left(t+(j+\gamma) \Delta T_{c}\right) d t \\
& \approx \begin{array}{cc}
n(1-|\gamma| \Delta), & j=i \text { and }|\gamma|<1 / 2 \\
\sqrt{n}, & |(j+\gamma)-i| \geq \frac{1}{\Delta} \text { and }|\gamma|<1 / 2
\end{array}
\end{aligned}
$$

is the partial crosscorrelation of the incoming and the local PN waveforms [8]. Thus, when $\psi=1, Y_{n}$ is a non-central chisquared r.v. with p.d.f. given by

$f_{Y_{n}}\left(y_{n}\right)=\frac{1}{2 \sigma_{n}^{2}} \exp \left(-\frac{y_{n} / \sigma_{n}^{2}+\lambda_{n} / \sigma_{n}^{2}}{2}\right) I_{0}\left(\sqrt{\frac{\lambda_{n}}{\sigma_{n}^{2}} \frac{y_{n}}{\sigma_{n}^{2}}}\right)_{(2.5)}$ where $\lambda_{n}=A_{0}^{2} T_{c}^{2} S_{n}^{2} / 4$ and $I_{0}(\bullet)$ is the modified Bessel function of order zero.

When $\psi$ is a Ricean fading r.v., things are more complicated. Conditioning on the chi-squared r.v. $\xi$ defined as $\xi=\psi^{2}, Y_{n}$ is conditionally a chi-squared r.v. The p.d.f. of $Y_{n}$ is therefore equal to

$$
f_{Y_{n}}\left(y_{n}\right)=\int_{0}^{\infty} f_{Y_{n}} l \xi\left(y_{n} \mid \xi\right) f(\xi) d \xi
$$

By deriving the identity [9]

$$
\sum_{k=0}^{\infty} \sum_{j=0}^{\infty} a^{k} b^{j} \frac{(k+j) !}{(k !)^{2}(j !)^{2}}=e^{a+b} I_{0}(2 \sqrt{a b})
$$

we were able to evaluate (2.6) and show that $Y_{n}$ is also a chisquared r.v. with p.d.f. given in the same form as (2.5). The parameter $\lambda_{n}$ in (2.5) is now replaced by

$$
\lambda_{\mathrm{n}} \frac{\mathrm{r}}{1+\mathrm{r}}
$$

and $\sigma_{n}^{2}$ replaced by

$$
\sigma_{n}^{2} \frac{(1+r)+\left(\lambda_{n} / 2 \sigma_{n}^{2}\right)}{1+r}
$$

The channel fading thus has an effect on reducing the per-chip signal-to-noise ratio defined as

$$
\mathrm{SNR}=\frac{\mathrm{A}_{0}^{2} \mathrm{~T}_{\mathrm{c}}}{2 \mathrm{~N}_{0}}=\frac{\lambda_{\mathrm{n}}}{2 \sigma_{\mathrm{n}}^{2}} \frac{\mathrm{n}}{\mathrm{S}_{\mathrm{n}}^{2}}
$$

by a factor of $r /\left(1+r+\lambda_{1} / 2 \sigma_{n}^{2}\right)$.

The TSPRT can be regarded as a hybrid mixture of the SPRT and the FSST [8]. Letting $\alpha_{S P R T}=p_{0} \alpha_{d}$ and $\beta_{S P R T}=p_{1}$ $\beta_{d}$, where $\alpha_{d}$ and $\beta_{d}$ are the design false-alarm and miss probability, respectively, and $\mathrm{p}_{0}$ and $\mathrm{p}_{1}$ are some design constants in $[0,1]$, we can design the upper and lower thresholds for the SPRT part. Letting $\alpha_{\mathrm{FSST}}=\left(1-\mathrm{p}_{0}\right) \alpha_{d}$ and $\beta_{\mathrm{FSST}}=\left(1-\mathrm{p}_{1}\right) \beta_{\mathrm{d}}$, we can design the truncated sample number and the corresponding cut-off threshold for the FSST part. An example of these threshold values and several sample paths of the normalized decision process are depicted in Figure 2. In Figure 2, the adjusted upper and lower threshold values for the TSPRT are displayed where the fading parameter $r$ is equal to 10. Setting $\psi=0.46$ under $\mathrm{H}_{1}$, the signal was severely attenuated by the fading and the test had to continue until the truncation point of $n=710$; the final decision statistic $(=9.058)$ was then compared to the cut-off threshold $(=8.48)$ and a decision of $\mathrm{H}_{1}$ was recorded in this example. The other two trajectories in Figure 2 are sample paths for two typical scenarios under $\mathrm{H}_{1}$ and $\mathrm{H}_{0}$.

For the FSST, the false-alarm and miss probabilities can be written explicitly as:

$$
P_{\mathrm{FA}}=\mathrm{Q}\left(\sqrt{2 \mathrm{SNR}}, \sqrt{\tau / \sigma_{\mathrm{M}}^{2}}\right)
$$

and

$$
P_{\text {MISS }}=1-Q\left(\sqrt{2 M \text { SNR }(1-|\gamma| \Delta)^{2}}, \sqrt{\tau / \sigma_{M}^{2}}\right)
$$

respectively, where the $Q(\cdot, \bullet)$ function is given by

$$
\mathrm{Q}(\mathrm{x}, \mathrm{y})=\int_{\mathrm{y}}^{\infty} \mathrm{z} \mathrm{e}^{-\left(\mathrm{z}^{2}+\mathrm{x}^{2}\right) / 2} \mathrm{I}_{0}(\mathrm{xz}) \mathrm{dz}
$$
The threshold and the sample size of the FSST can be evaluated using these equations. For a given value of the design $\mathrm{P}_{\mathrm{FA}}$, we use $(2.10)$ to compute the normalized threshold, $\tau / \sigma_{M}^{2}$; then

(2.11) can be employed to solve for the required sample size $\mathbf{M}$ for the design PMISs.

\section{Acquisition with Data Modulation}

Assuming that data modulation is BPSK, we can write the received signal as

$r(t)=A_{0} d\left(t+i \Delta T_{c}\right) a\left(t+i \Delta T_{c}\right) \cos \left(\omega_{0} t+\theta\right)+n(t)$ where $d(t)$ is the \pm -valued data waveform. Note that based on the results stated in Section II, extension of the analysis to include both modulation and fading is trivial. Now, under $\mathrm{H}_{0}$ (non-alignment), the statistics of $Y_{n}$ are unchanged. Under $H_{1}$ (alignment), we may assume that $n<N$, where $\mathrm{N}$ is the period of the PN sequence, and the probability of having a data boundary within the integration interval $\left(0, n_{c}\right)$ to be $n / N$. Then we compute

$$
\begin{aligned}
S_{\mathbf{n}}= & \pm[(2 \mathrm{i}-\mathrm{n})(1-|\gamma| \Delta) \\
& \left.\left.+|\gamma| \Delta \mid \sum_{\mathrm{k}=0}^{\mathrm{i}-1} c_{\mathrm{k}} \mathrm{c}_{\mathrm{k}+\operatorname{sgn}(\gamma)}-\sum_{\mathbf{k}=\mathbf{i}}^{\mathrm{n}-1} c_{k} c_{k}+\operatorname{sgn}(\gamma)\right\}\right]
\end{aligned}
$$$$
\approx \pm(2 \mathrm{i}-\mathrm{n})(1-|\gamma| \Delta)
$$

where $c_{k}$ is the PN chip value and the data boundary location" $i$ " is modeled as a discrete r.v. on $\{0,1, \ldots, n-1\}$. Eqn. (3.2) has been obtained by assuming that there is a change of polarity at the $\mathrm{i}$-th chip. The \pm sign results from the direction of polarity changes, i.e., from + to - and from - to + . The p.d.f. of $Y_{n}$ under $\mathrm{H}_{1}$ is then given by

$$
\begin{aligned}
f_{Y_{n}}\left(y_{n} \mid H_{1}\right)=\left(1-\frac{n}{2 N}\right) \frac{1}{2 \sigma_{n}^{2}} & \exp \left(-\frac{y_{n} / \sigma_{n}^{2}+\lambda_{n} / \sigma_{n}^{2}}{2}\right) \\
\cdot & I_{0}\left(\sqrt{\frac{\lambda_{n}}{\sigma_{n}^{2}} \frac{y_{n}}{\sigma_{n}^{2}}}\right)
\end{aligned}
$$




$$
\begin{aligned}
+\frac{1}{2 N} \sum_{i=0}^{n-1} \frac{1}{2 \sigma_{n}^{2}} & \exp \left(-\frac{y_{d} / \sigma_{n}^{2}+((n-2 i) / n)^{2} \lambda_{n} / \sigma_{n}^{2}}{2}\right) \\
& \cdot I_{0}\left(\frac{|n-2 i|}{n} \sqrt{\frac{\lambda_{n} \frac{y_{n}}{\sigma_{n}^{2}}}{\sigma_{n}^{2}}}\right)
\end{aligned}
$$

where $\lambda_{n} / \sigma_{n}^{2}=2 n(S N R)(1-|\gamma| \Delta)^{2}$. This $Y_{n}$ of (3.3) is no

longer a chi-squared r.v., and the effect of data modulation under $\mathrm{H}_{1}$ is less straightforward to estimate. As $n$ increases, the crosscorrelation value $S_{n}$ increases in magnitude (positive effect) but the probability of seeing a data boundary also increases (negative effect). If we consider the average value of $\lambda_{n} / 2 \sigma_{n}^{2}$, we get

$$
\begin{aligned}
\frac{\bar{\lambda}_{n}}{2 \sigma_{n}^{2}} & =\frac{\lambda_{n}}{2 \sigma_{n}^{2}}\left[\left(1-\frac{n}{2 N}\right)+\frac{1}{2 N} \sum_{i=0}^{n-1}\left(\frac{2 i-n}{n}\right)^{2}\right] \\
& =\frac{\lambda_{n}}{2 \sigma_{n}^{2}}\left[1-\frac{n}{3 N}+\frac{1}{3 N n}\right] \\
& >\frac{\lambda_{n}}{2 \sigma_{n}^{2}} \frac{2}{3}
\end{aligned}
$$

so the effective average SNR may be reduced by at most a factor of $1 / 3$. If we let $n / N \approx 20 \%$ (see typical numerical results below), the change in the effective SNR due to data modulation is only around $10 \log _{10} 14 / 15 \approx-0.3 \mathrm{~dB}$.

\section{Numerical Results}

In this section, we set $\Delta=1 / 2$, chip $\mathrm{SNR}=-10 \mathrm{~dB}, \gamma=$ $0.5, p_{0}=p_{1}=0.5$, and $N=1023$. The phase of the incoming PN signal is assumed to be zero, i.e., $i=0$ in (2.1). Unless otherwise specified, the design false-alarm probability $\left(\alpha_{d}\right)$ and the design miss probability $\left(\beta_{d}\right)$ are both set to 0.01 .

In Figures 3.a and 3.b, we show the effect of fading on the TSPRT. Figure 3.a depicts the power function (probability of deciding $\mathrm{H}_{1}$ ), while Figure 3.b sketches the average sample number (ASN) vs. the phase offset parameter $|j+\gamma|$. When $\mid j+$ $\gamma \mid \leq 0.5$, the system is under $H_{1}$; on the other hand, when $\mid j+\gamma$ $1 \geq 2.0$, the system is under $\mathrm{H}_{0}$. Our results for the TSPRT are obtained through simulations and averaging. In the simulations, the p.d.f. of the fading variable $\psi$ in (2.1) is divided into 25 segments of equal areas. The mean value of each segment is used in the simulations. Results from these 25 cases are then averaged to obtain the final data. It can be seen from Figure 3.a that the detection probability can degrade significantly with fading, especially when $r$ is small. As an example, at $r=10, P_{d}$ is reduced from 0.99 to 0.93 . Using the new p.d.f. derived in Section II to account for the fading, the upper and lower thresholds are adjusted accordingly and the power of the test under $\mathrm{H}_{1}$ can be restored. The results for the adjusted threshold cases are also included in these figures. Both Figures 3.a and 3.b show that the power and the ASN under $\mathrm{H}_{0}$ are unaffected by the channel fading. This fact is expected because under $\mathrm{H}_{0}$, the out-of-synchronization SS signal appears like noise to the decision circuit. However, the price to pay for achieving the desired power are the larger ASN values, in particular the larger ASN value under $H_{0}$. For example, under $H_{0}$ with $r=10$, the ASN is increased from 158 to 308 . Since most states in testing are under $\mathrm{H}_{0}$, we should keep the ASN value under $\mathrm{H}_{0}$ small. Our results suggest that it may not be worthwhile to adjust the thresholds even if the fading level, $r$, can be estimated. The loss in the detection probability due to fading appears to lie within tolerable bounds for moderate values of $r$, furthermore, the ASN does not change drastically with fading. For moderate values of $r(\approx 10)$, the performance of the TSPRT is rather robust to fading impairment. For small values of $r(\approx 1)$, the TSPRT considered in this paper may not perform.

In Figures 4.a and 4.b, we show the effect of modulation on the TSPRT. We have modeled the data boundary location " $i$ " in (3.2) as a discrete uniform variable and the results are obtained by averaging over " $i$ ". The effective $(\alpha, 1-\beta)$ values are now changed from $(0.0059,0.991)$ to $(0.0061$, 0.948 ) by the data modulation. The ASN was essentially unchanged. To probe further, we also present additional results of the modulation effects on TSPRT under $\mathrm{H}_{1}$ in Figures 5.8 and 5.b. The value of the design false-alarm probability is fixed $\left(\alpha_{d}=0.01\right)$, but the design detection probability $P_{d}\left(=1-\beta_{d}\right)$ is varying. In Figure 5.a, the effective detection probability of the TSPRT is plotted against the design detection probability. It can be seen that when there is no modulation, the TSPRT can achieve the design operation points; in fact, our TSPRT is overdesigned, especially at smaller design detection probability values. On the other hand, when there is modulation, the effective detection probability is decreased, as expected. We also compare the corresponding ASN values (under $\mathrm{H}_{1}$ ) of the TSPRT in Figure 5.b. As is shown in this figure, the ASN of the TSPRT does not differ significantly when modulation is added. Although it is possible to restore the full desired power of the TSPRT by adjusting the thresholds and sample size via (3.3), our data indicates that the relatively minute loss in performance of the test due to data modulation may not warrant such an effort.

For comparison purposes we show the effect of data modulation on the FSST in Figures 6.a and 6.b. Fig. 6.a shows the power of FSST with or without data modulation. Under $\mathrm{H}_{1}$, FSST's detection probability is decreased from 0.99 to 0.921 by data modulation. The false-alarm probability is not changed. Comparing with Fig. 4.a, we see that data modulation has less an effect on the detection probability for TSPRT than FSST Fig. 6.b shows the variation of the effective detection probability with the design detection probability and fixed $\alpha$. The curves are fairly linear, but they are below the ideal characteristic which would be a line of slope 1 passing through the origin. The degree to which the curves are offset from the ideal curve decreases as $\alpha$ increases. As we increase $\alpha$, the test termination point decreases and data modulation becomes less devastative to the detection probability. One may use the model of (3.3) to adjust the threshold and restore the test power in many cases; a detailed study can be found in [11]

\section{Conclusions}

The power and ASN of the TSPRT and the FSST for noncoherent PN sequence acquisitions have been investigated The effects of Ricean fading and data modulations on the tests were considered. It was found that the p.dff of the decision statistic preserved the chi-squared distribution when Ricean fading was added to the channel. Channel fading in effect induced a loss of the SNR, but the design power of the tests can be restored by making suitable threshold adjustments and increasing the sample size. The power of the TSPRT decreased significantly with severe fading, but the corresponding ASN values did not increase drastically. For moderate fading, the performance of the TSPRT was found to be robust to the fading impaiment. Comparing with FSST, the detection probability of TSPRT was less sensitive to data modulation. 


\section{Reference}

[1] A. Polydoros and C. L. Weber, "A unified approach to serial search spread-spectrum code acquisition - Part I: general theory, Part II: a matched-filter receiver," IEEE Trans. Commun., Vol. COM-32, pp. 542-560, May 1984.

[2] D. Hall and C. L. Weber, "Two methods for noncoherent acquisition of DS waveforms," Proc. MILCOM Conf., pp. 13.7.1-13.7.5, 1987.

[3] Y. T. Su and C. L. Weber, "A class of sequential tests and its applications," IEEE Trans. Commun., Vol. COM-38, pp.165-171, Feb. 1990.

[4] K. K. Chawla and D. V. Sarwate, "Coherent acquisition of PN sequences for DS/SS systems using a random sequence model and the SPRT," Proc. 1990 Conf. on Information Sciences and Systems, Princeton Univ., New Jersey, pp. 52-57, March 1990.

[5] Y. H. Lee and S. Tantaratana, "Acquisition of PN sequences for DS/SS systems using a truncated sequential probability ratio test," Journal of the Franklin Institute, Vol. 328, pp. 231-248, 1991.

[6] E. Sourour, S. C. Gupta and W. Refai, "Direct sequence spread spectrum serial acquisition in a nonselective and frequency selective Ricean fading channel," Proc. MILCOM Conf., pp. 5.7.1-5.7.5, 1990.

[7] U. Cheng, W. J. Hurd and J. I. Statman, "Spread spectrum code acquisition in the presence of Doppler shift and data modulation," IEEE Trans. Commun., Vol. COM-38, pp. 241-250, Feb. 1990.

[8] S. Tantaratana and A. W. Lam, "Noncoherent sequential acquisition for DS/SS systems," Proc. Twenty-Ninth Annual Allerton Conf. on Commun., Control, and Computing, University of Illinois at Urbana-Champaign, Oct. 1991.

[9] S. Tantaratana, A. W. Lam and P. J. Vincent, "Noncoherent sequential acquisition of PN sequences for DS/SS communications with/without channel fading," submitted for publication.

[10] D. Divsalar and M. K. Simon, "The design of trelliscoded MPSK for fading channel: performance criteria," IEEE Trans. Commun., Vol. COM-36, pp. 1004-1012, Sep. 1988.

[11] P. J. Vincent, "Effects of fading and data modulation on noncoherent m-sequence acquisition schemes," M.S. thesis, Naval Postgraduate School, Monterey, CA, March 1992.

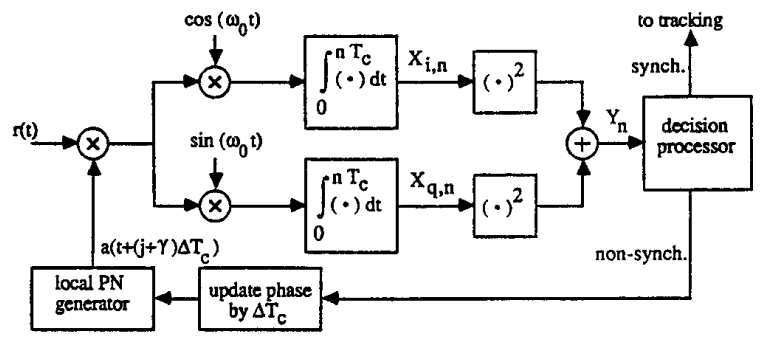

Figure 1 Block diagram of noncoherent serial acquisition scheme.

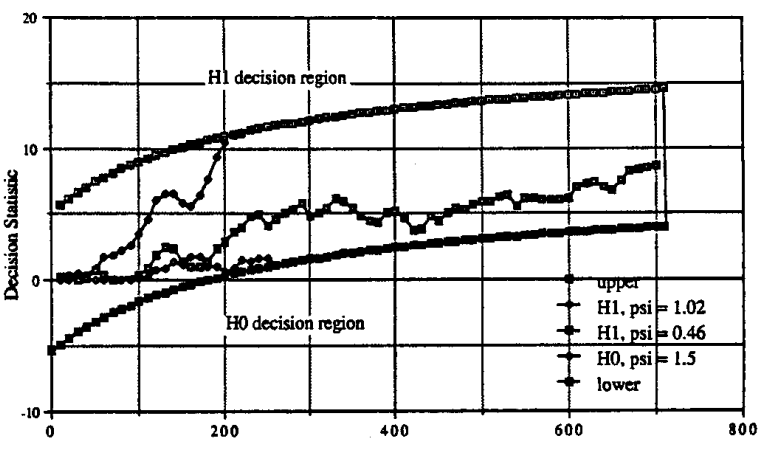

Figure 2 Sample paths of the TSPRT in Ricean fading channel, $r=10$.

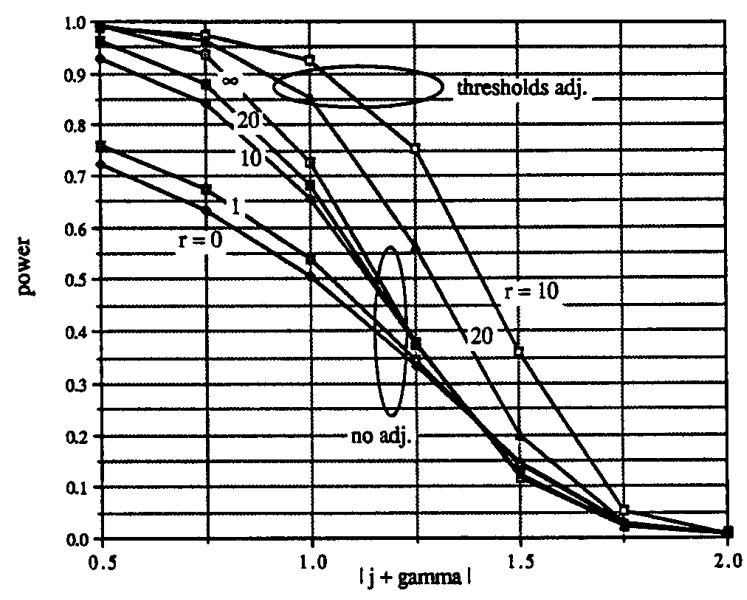

(a)

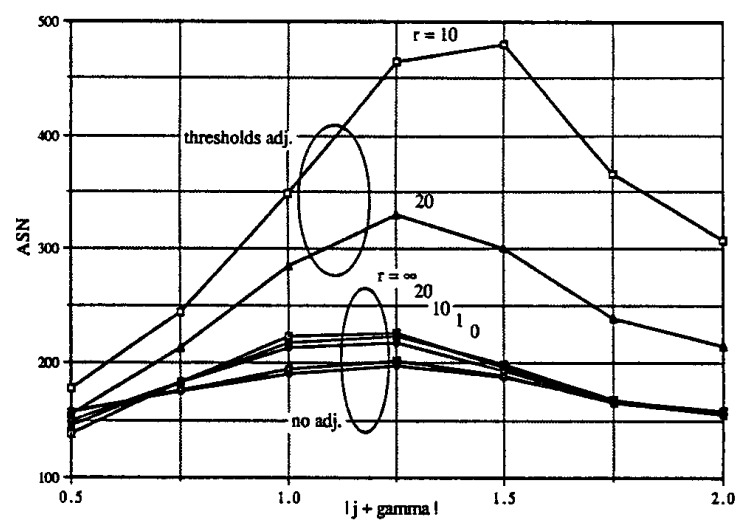

(b)

Figure 3 Performance of TSPRT with Ricean fading. (a) Power. (b) ASN. 


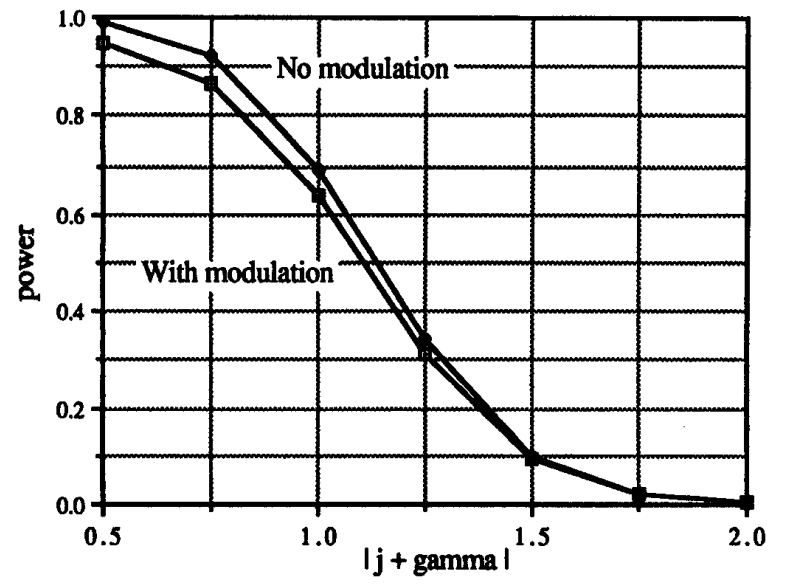

(a)

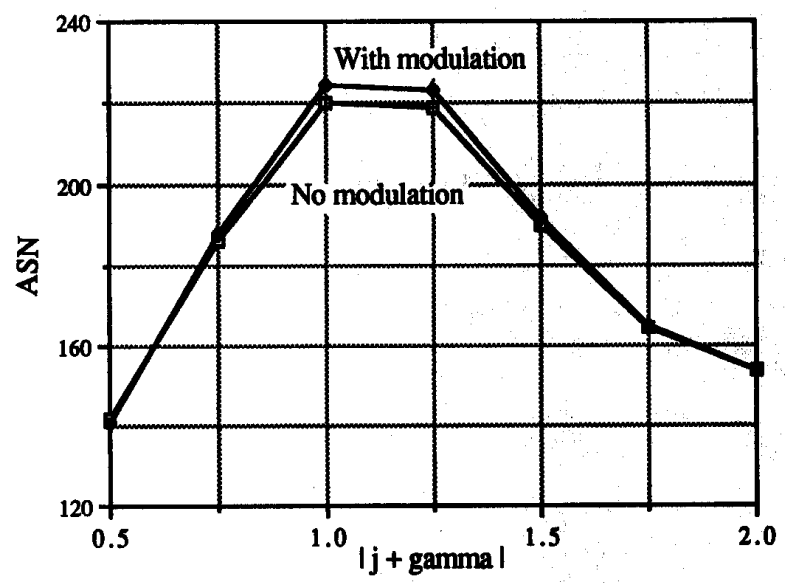

(b)

Figure 4 Performance of TSPRT with data modulation. (a) Power. (b) ASN.

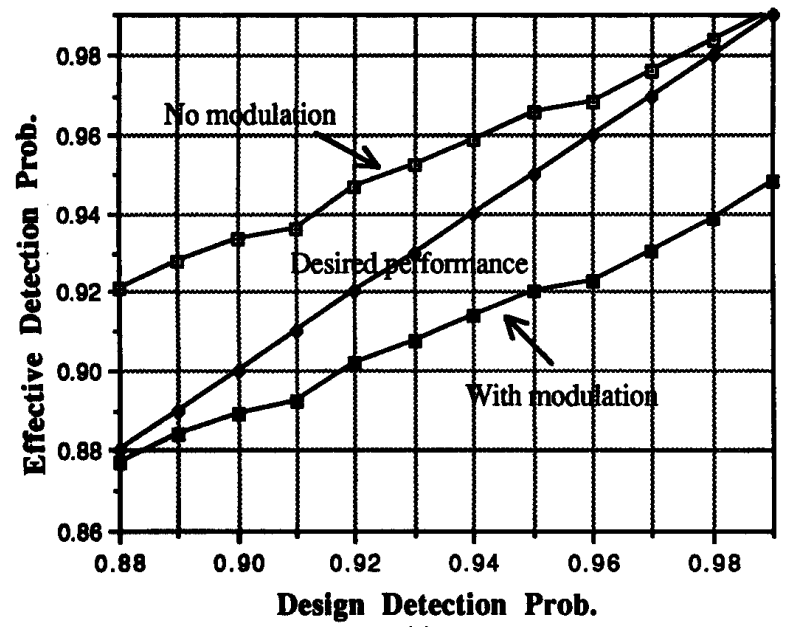

(a)

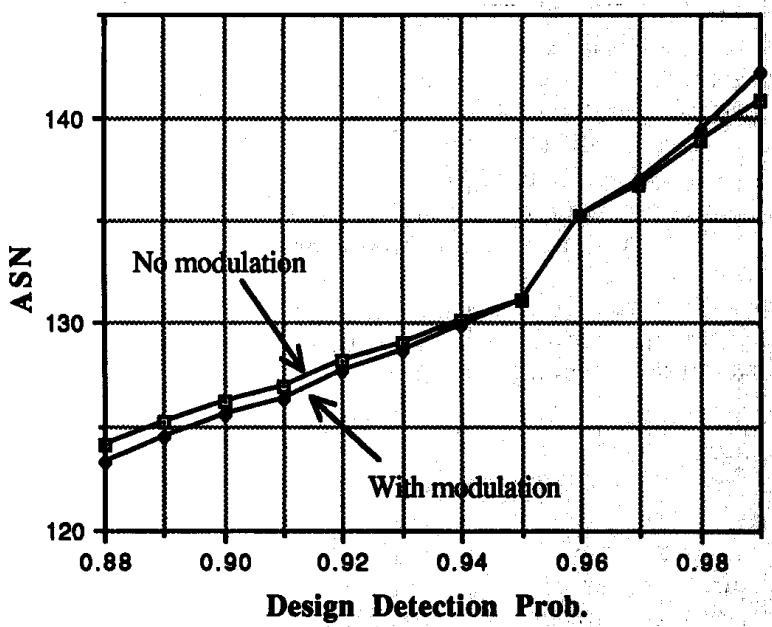

(b)

Figure 5 Effect of modulation on the performance of TSPRT, $\alpha_{d}=0.01$. (a) Effective detection probability. (b) ASN.

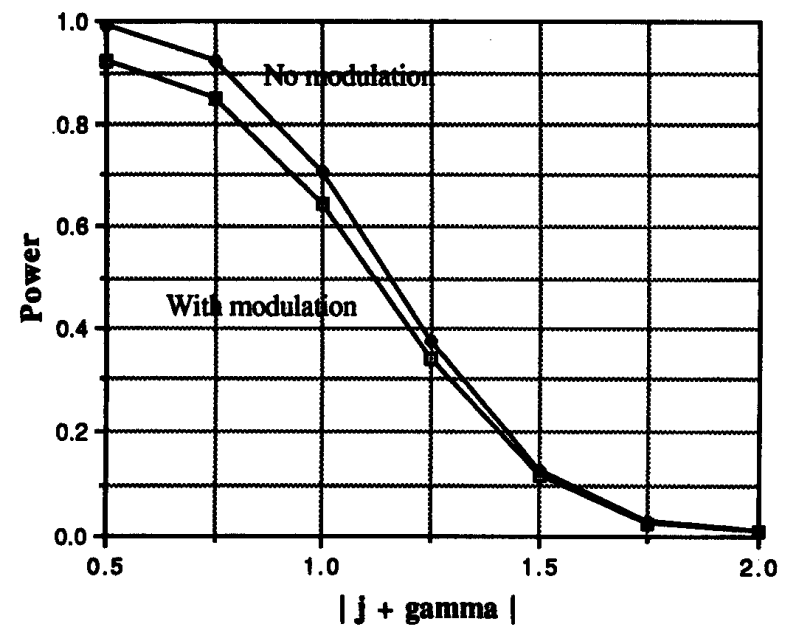

(a)

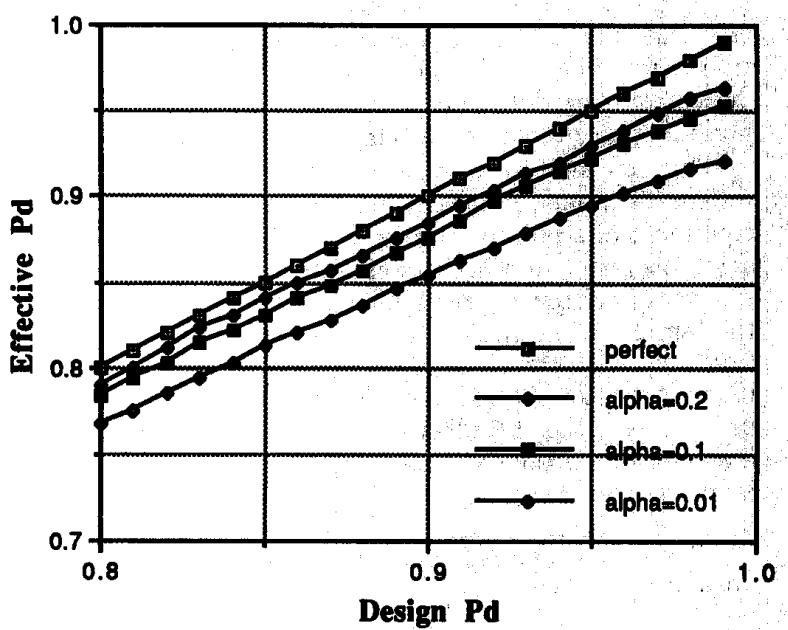

(b)

Figure 6 Performance of FSST with data modulation. (a) Power. (b) Effective vs. design detection probabilities at fixed $\alpha$. 17.1 .5 